\title{
On the estimation of temporal changes of snow water equivalent by spaceborne SAR interferometry: a new application for the Sentinel-1 mission
}

\author{
Vasco Conde $^{1}$, Giovanni Nico ${ }^{2 *}$, Pedro Mateus ${ }^{1}$, João Catalão ${ }^{1}$, Anna Kontu ${ }^{3}$, Maria Gritsevich ${ }^{4,5,6}$ \\ ${ }^{1}$ Instituto Dom Luiz (IDL), Universidade de Lisboa, 1749-016 Lisboa, Portugal. \\ ${ }^{2}$ Consiglio Nazionale delle Ricerche, Istituto per le Applicazioni del Calcolo (CNR-IAC), 70126 Bari, Italy. \\ ${ }^{3}$ Finnish Meteorological Institute (FMI), Sodankylä, Finland. \\ ${ }^{4}$ Department of Physics, P.O. Box 64, FI-00014 University of Helsinki, Finland. \\ ${ }^{5}$ Institute of Physics and Technology, Ural Federal University, Ekaterinburg, Russia. \\ ${ }^{6}$ Dorodnicyn Computing Centre, Russian Academy of Sciences, Moscow, Russia. \\ ${ }^{*}$ Corresponding author. E-mail: g.nico@ba.iac.cnr.it
}

\begin{abstract}
In this work we present a methodology for the mapping of Snow Water Equivalent (SWE) temporal variations based on the Synthetic Aperture Radar (SAR) Interferometry technique and Sentinel-1 data. The shift in the interferometric phase caused by the refraction of the microwave signal penetrating the snow layer is isolated and exploited to generate maps of temporal variation of SWE from coherent SAR interferograms. The main advantage of the proposed methodology with respect to those based on the inversion of microwave SAR backscattering models is its simplicity and the reduced number of required in-situ SWE measurements. The maps, updated up to every 6 days, can attain a spatial resolution up to $20 \mathrm{~m}$ with sub-centimetre $\triangle \mathrm{SWE}$ measurement accuracy in any weather and sun illumination condition. We present results obtained using the proposed methodology over a study area in Finland. These results are compared with in-situ measurements of $\triangle \mathrm{SWE}$, showing a reasonable match with a mean accuracy of about $6 \mathrm{~mm}$.
\end{abstract}

Keywords: Snow Water Equivalent (SWE); Synthetic Aperture Radar (SAR); SAR interferometry (InSAR); Sentinel-1.

\section{INTRODUCTION}

The Snow Water Equivalent (SWE) is the quantity of water stored in the snowpack. Its measurement is important for predicting the water supply at the snowmelt time, useful for both assessing the energy potential of snow in hydroelectric power plants and the flood risk in valleys and plain areas crossed by rivers. Furthermore, the seasonal snow cover is an important component in land surface climate since it causes a seasonal variation in surface albedo, and it has a low thermal conductivity, so it insulates the soil surface from large, rapid temperature fluctuations. The monitoring of snow cover with a high spatial and temporal resolution over large areas can provide useful information to Numerical Weather Models (NWMs) since it can help to set dynamic boundary conditions based on the land cover. Estimates of the SWE can be extracted by using remote sensing techniques. Different remote sensing techniques have been used to estimate the SWE. As an example, Snow-Covered Area (SCA) maps provided by the Moderate Resolution Imaging Spectroradiometer (MODIS) have been used in SWE models (see e.g. Bavera and De Michele (2009), Guan et al. (2013)).

However, visible and near-infrared sensors are affected by cloud cover that can hinder the possibility to image the terrain surface. Radiometers provide information on snow at a very low spatial resolution (about $25 \mathrm{~km}$ ) and have difficulties in both deep and shallow snow conditions (Takala et al., 2011). A new microwave spaceborne mission has been presented to the European Space Agency (ESA) for the high resolution mapping of SWE and of its temporal variations to study climate changes and their impact on the environment (Rott et al., 2012a; Rott et al., 2012b). However, the snow accumulation and melt are highly variable in space and time. This requires detailed field measurements to correctly model the snow variability on catchment scale (Krajči et al., 2016). Synthetic Aperture Radar (SAR) can provide high resolution maps of snow properties over wide areas, in all-weather and day/night conditions. SAR images are currently acquired by different spaceborne missions at different microwave frequencies, X-band (COSMO-SkyMed and TerraSAR-X), C-band (Sentinel-1 and RADARSAT-2) and L-band (ALOS-2) using different polarization channels $(\mathrm{HH}$, $\mathrm{HV}, \mathrm{VV}$, where e.g. HV means that the SAR image is obtained by illuminating the scene with H-polarized microwave signal and receiving the $\mathrm{V}$-polarized component of the backscattered signal). SAR images have been used to study the snow physical properties based on their capability to penetrate the snowpack or being absorbed when the snow is melting (Malnes and Guneriussen, 2002; Nagler and Rott, 2000; Pivot, 2012; Sun et al., 2015). The SWE is defined in terms of snow density and depth. The first quantity can be estimated by L-band SAR images (Shi and Dozier, 2000a). Although the L-band radar signal passes easily through dry snow, snow density affects the radar backscattering coefficient. The snow density is estimated using L-band VV and $\mathrm{HH}$ measurements and a numerical model to compute the backscattering coefficients (Shi and Dozier, 2000a). Furthermore, the $\mathrm{C}$ and X-bands have been used to retrieve the snow depth (Shi and Dozier, 2000b). The algorithm does not require a priori knowledge of the subsurface dielectric and roughness properties. A numerical model is also used to invert $\mathrm{C}$ and $\mathrm{X}$-band SAR images to estimate the snow depth (e.g. see Longepe et al. (2009), Shi and Dozier (2000b)). Usually, this requires a large amount of in-situ measurements to accurately estimate the relationship between the radar cross section and the snow properties.

In this manuscript, we investigate the use of SAR interferometry (InSAR) to map SWE over large areas using spaceborne C-band Sentinel-1 SAR data, at the light of their short revisiting time of six days, large spatial coverage and high spatial resolution of 20 meters. Furthermore, ESA is providing Sentinel-1 data using a free-access policy. The main motivation of this work is to understand if the use of InSAR technique can reduce 
the amount of in-situ SWE measurements while keeping all the other advantages of SAR images, mainly its high spatial resolution. In fact, SWE estimation methods based on SAR amplitude images require many in-situ SWE measurements to invert the microwave SAR backscattering model.

Guneriussen et al. (2001) presented a theory to explain the presence of fringes in SAR interferograms acquired over areas covered by dry snow. They interpreted the interferometric phase in terms of a possible temporal change in the SWE. However, the authors pointed out that in-situ meteorological observations were needed to distinguish the phase contributions due to the temporal change of SWE from the propagation effects in atmosphere. The physical principle used by SAR interferometry is that of phase delay due to propagation in a nondispersive medium. This implies that the snow is supposed to be dry to allow the propagation of the SAR signal. The fact that the SWE estimation is based on the measurement of a phase delay implies that phase contributions due to terrain morphology and propagation in the atmosphere should be properly modeled and corrected. For instance, a precise Digital Elevation Model (DEM) of the area is used to model and remove the phase due to topography modulation of the interferometric signal. Critical is the mitigation of the atmospheric phase delay since it is characterized by a low spatial frequency fringe pattern affecting the whole interferogram. These characteristics could be similar to the spatial distribution of SWE in the winter season and for this reason it is very important to remove the phase component due to propagation in atmosphere. The atmosphere's phase delay can be modeled by using external data such as NWMs (Mateus et al., 2015; Nico et al., 2011) or delay measurements provided by Global Navigation Satellite System (GNSS) receivers or passive satellite sensors, such as MERIS in the past or MODIS for current studies (Mateus et al., 2010). Concerning the presence of terrain displacements, due to different geological phenomena or to the human activity, it is assumed that they are negligible within the time interval between the acquisitions of the master and slave SAR images used to generate the interferograms. The proposed methodology provides a direct estimate of the snow depth which is then used to derive the SWE.

Even if it is no longer needed to invert a backscattering model relating SAR data to in-situ measurements of SWE, as in the case of the estimation techniques based on the SAR backscattering coefficients, the knowledge of the SWE in a few points is still needed to calibrate InSAR data. The main reason for this is related to the interferometric processing of SAR data. In fact, phase measurements are always relative to a reference point. In space geodesy applications of SAR interferometry, the reference point is a stable target which has no displacement in the time interval between the master and slave images or a target with known displacement rate. In this paper, the reference points correspond to sites where the SWE is provided by in-situ measurements. Absolute estimates of the SWE can be obtained by building a time series of SAR images sharing the same acquisition parameters, with the first image during the summer season when the SWE is zero. An important novelty of this work is the use of a numerical weather model to estimate the 3-D fields of the most relevant atmospheric physical parameters (including atmosphere's temperature, relative humidity and total pressure) with a $1 \mathrm{~km}$ spatial resolution and a variable vertical resolution from the Earth surface up to an altitude corresponding to $10 \mathrm{hPa}$. These parameters are used to model the microwave phase delay in troposphere. The proposed methodology is applied in an experiment to estimate the temporal variation of SWE, from December 29, 2015 to
January 22, 2016, over a large area in the northeastern part of Finland.

\section{STUDY AREA AND DATASET}

The study area is located at the northeastern border of Finland $\left(66^{\circ} \mathrm{N}, 28^{\circ} \mathrm{E}\right)$ as shown in Figure 1. It partially covers the three Finnish regions of Kainuu, Northern Ostorbothnia, and Lapland. The small portion of the Russian territory which is in the Sentinel-1's footprint has been removed by means of a mask. It spans an area of about $22000 \mathrm{~km}^{2}$ with an average altitude of $250 \mathrm{~m}$ above the sea level. The minimum and maximum altitudes of the study area are $110 \mathrm{~m}$ and $511 \mathrm{~m}$ a.s.1., respectively. The area is characterized by a moderate topography, with an altitude standard deviation of $55 \mathrm{~m}$, and a seasonally snow covered surface in the winter season.

Three consecutive C-band ESA Sentinel-1 SAR images, acquired between December 2015 and January 2016 in Interferometric Wide (IW) swath mode along a descending pass, have been used. Each image has a dual polarization $\mathrm{VV}+\mathrm{VH}$, a $32^{\circ}-$ $43^{\circ}$ incidence angle, and a spatial resolution of $5 \mathrm{~m} \times 20 \mathrm{~m}$. Two consecutive interferograms have been generated from the SAR images. The interferograms were generated by keeping the temporal baseline at the lowest possible value of twelve days. Each interferogram was corrected for the topographic phase contribution, using a DEM with a spatial resolution of 10 $\mathrm{m}$ and a vertical accuracy of $1.4 \mathrm{~m}$, created by the National Land Survey of Finland (see Figure 1), and then multilooked. Table 1 summarizes the acquisition dates of the master and slave

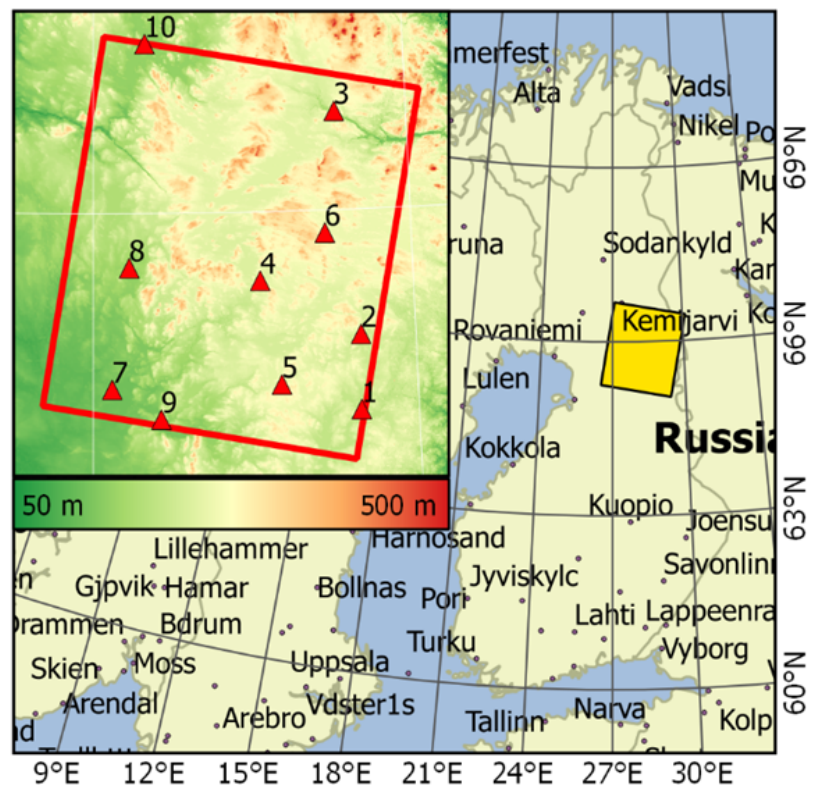

Fig. 1. Location of the study area (yellow rectangle) on the Scandinavian Peninsula map. The Digital Elevation Model (DEM) of the area is reported in the inset on the upper-left corner, where the ten locations of in-situ SWE measurements (red triangles) and the footprint of the SAR images (red outline) are overlaid.

Table 1. Master and slave acquisition dates, temporal and perpendicular spatial baselines of the interferograms.

\begin{tabular}{|l|c|c|c|}
\hline $\begin{array}{l}\text { Master image } \\
\text { acquisition date }\end{array}$ & $\begin{array}{c}\text { Slave image } \\
\text { acquisition date }\end{array}$ & $\begin{array}{c}\text { Temporal } \\
\text { baseline (days) }\end{array}$ & $\begin{array}{c}\text { Perpendicular } \\
\text { baseline (m) }\end{array}$ \\
\hline $2015-12-29$ & $2016-01-10$ & 12 & 43.60 \\
\hline $2016-01-10$ & $2016-01-22$ & 12 & 55.45 \\
\hline
\end{tabular}




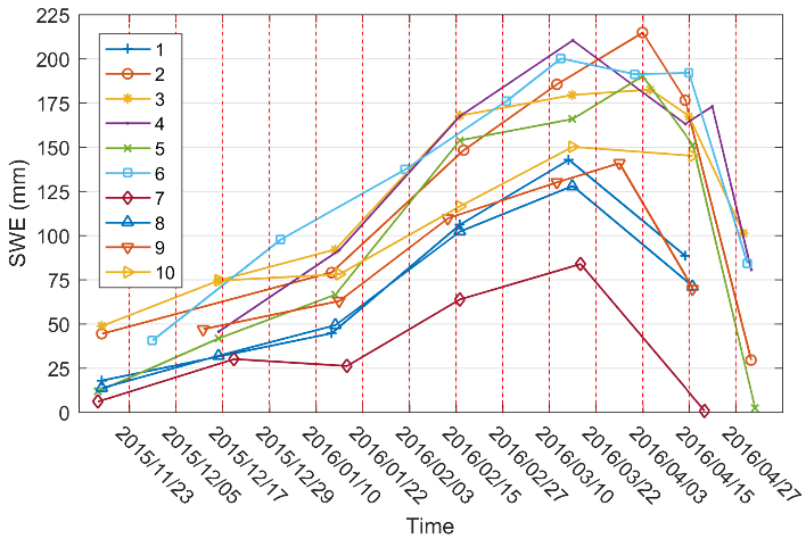

Fig. 2. SWE measurements for each in-situ location from November 2015 till April 2016. Sentinel-1 SAR acquisition dates are represented by vertical dashed lines. For the acquisition dates of master and slave SAR images (Table 1) SWE values have been estimated by $2^{\text {nd }}$ order polynomial regression.

Sentinel-1 SAR images, the temporal and perpendicular baselines of the corresponding interferograms.

The in-situ SWE data are measured at ten different locations scattered across the study area, as reported in Figure 1. The time series of SWE values, shown in Figure 2, are from snow course measurements made by the Finnish Environment Institute (SYKE) (available at http://www.syke.fi/enUS/Open information). The measurement, made along $2 \mathrm{~km}$ long snow courses, have been carried out over different land cover categories, so that an average calculated from individual measurements gives a representative SWE value for the location.

The course is typically measured once per month using manual snow depth probe and snow tube (Leppänen et al., 2016).

Cloud-free Sentinel-2 MSI Natural Colors images and a recently updated Corine map of the study area have been used to get information about land use and cover around each in-situ measurement location (see Figure 3).

It is worth noting that, as plotted in Figure 2, the acquisition dates of in-situ SWE measurement and Sentinel-1 data do not coincide. As a consequence, the in-situ reference SWE values at the acquisition times of master and slave images, used to generate the interferograms (see Table 1), have been obtained by a 2-nd order polynomial regression from data acquired from November 23, 2015 till March 10, 2016 (see Figure 2). In-situ measurements acquired in April have not been used as it has not been possible to generate coherent SAR interferograms for that period.

\section{METHODOLOGY}

The interferometric phase, computed as the complex product of two satellite SAR images, contains different contributions. The most significant ones are: 1) topography, 2) terrain displacement, 3) atmospheric phase delay, 4) contribution due to inaccurate knowledge of satellite orbits and hence of the interferometric spatial baseline. All these contributions can be identified and removed or at least mitigated. The topographic contribution is removed using a DEM of the area. The atmospheric contribution can be modeled and mitigated by using external data such as Numerical Weather Model (NWM) output or permanent GPS receivers and meteorological stations. The phase contribution due to satellite orbits errors are easily identified since it gives rise to phase ramps in the interferogram which can be removed. Contribution due to terrain displace- ment (e.g. due to landslides, earthquakes, and subsidence) are not easily identified since they usually affect small areas and are related to geological phenomena and/or human activity. In this work we assumed they are negligible over the temporal interval of twelve days. We assume that the residual phase is related to the propagation of microwaves into the snow layer.

When isolated from the contributions listed above, the interferometric phase due to the propagation into the snow layer can be used to estimate the temporal changes of SWE. In fact, due to the dielectric contrast at air-snow interface, the radar signal is refracted when it impinges on the snow layer, resulting in a longer path $\left(\Delta R_{S}\right)$ when compared with the linear path $\left(\Delta R_{A}\right)$ as sketched in Figure 4. A relationship can be found between the variation of snow depth and the measured interferometric phase (Guneriussen et al., 2001):

$$
\Delta \phi_{S}=\frac{4 \pi}{\lambda_{0}} \Delta Z_{S}\left(\cos \theta_{i}-\sqrt{\epsilon-\sin ^{2} \theta_{i}}\right)
$$

where $\Delta \phi_{S}$ is the interferometric phase contribution due to the 2-way propagation into the snow layer, measured as the phase difference between two SAR images acquired at two different times (master and slave SAR images). The other parameters of the equation are: the radar wavelength $\lambda_{0}$, the surface incidence angle $\theta_{i}$, the snow depth temporal variation $\Delta Z_{S}$, and the snow relative permittivity $\epsilon$ which depends on the snow density $\rho_{S}$. The SWE is defined as:

$$
\operatorname{SWE}\left(\rho_{S}\right)=\frac{\rho_{S}}{\rho_{W}} Z_{S}
$$

where $\rho_{W}$ is the liquid water density. From Equations (1) and (2) the following relationship can be found between the temporal variation of SWE and the interferometric phase:

$$
\Delta \operatorname{SWE}=\operatorname{SWE}\left(t_{s}\right)-\operatorname{SWE}\left(t_{m}\right)=\frac{\lambda_{0}}{2 \pi} \cdot \frac{1}{\alpha\left(1.59+\theta_{i}^{\frac{5}{2}}\right)} \Delta \phi_{s}
$$

where $t_{m}$ and $t_{s}$ are, respectively, the acquisition times of the master and slave SAR images and $\alpha \cong 1$ is a parameter slightly depending on the incidence angle $\theta_{i}$ (Leinss et al., 2015). The local incidence angle is estimated from the DEM, computing the local slope and aspect angles, and the spaceborne SAR look angle. Using the Sentinel-1 SAR wavelength $\lambda_{0}=5.5 \mathrm{~cm}$, a mean incidence angle of $\theta_{i}=37^{\circ}$ and $\alpha \cong 1$, Equation (3) can be re-written as:

$$
\Delta \operatorname{SWE}=\operatorname{SWE}\left(t_{s}\right)-\operatorname{SWE}\left(t_{m}\right)=0.0045 \Delta \phi_{S}
$$

This relationship says that since the phase variation $\Delta \phi_{s}$ measured by SAR interferometry is always within the basic interval $[-\pi, \pi] \mathrm{rad}$, the temporal variation of SWE can be estimated unambiguously, without the need of phase unwrapping, only in the interval $[-14.3,14.3] \mathrm{mm}$. It is worth noting that this interval depends on the local incidence angle and so in real conditions it slightly varies in space according to the local $\theta_{i}$.

A key point of the proposed methodology is the modeling of the atmospheric phase delay. In fact, the interferometric phase signal has two main contributions, after the correction of topography contribution, and in case of no significant terrain deformation within the time window between two SAR acquisitions, phase delays in atmosphere and the snow layer. If 

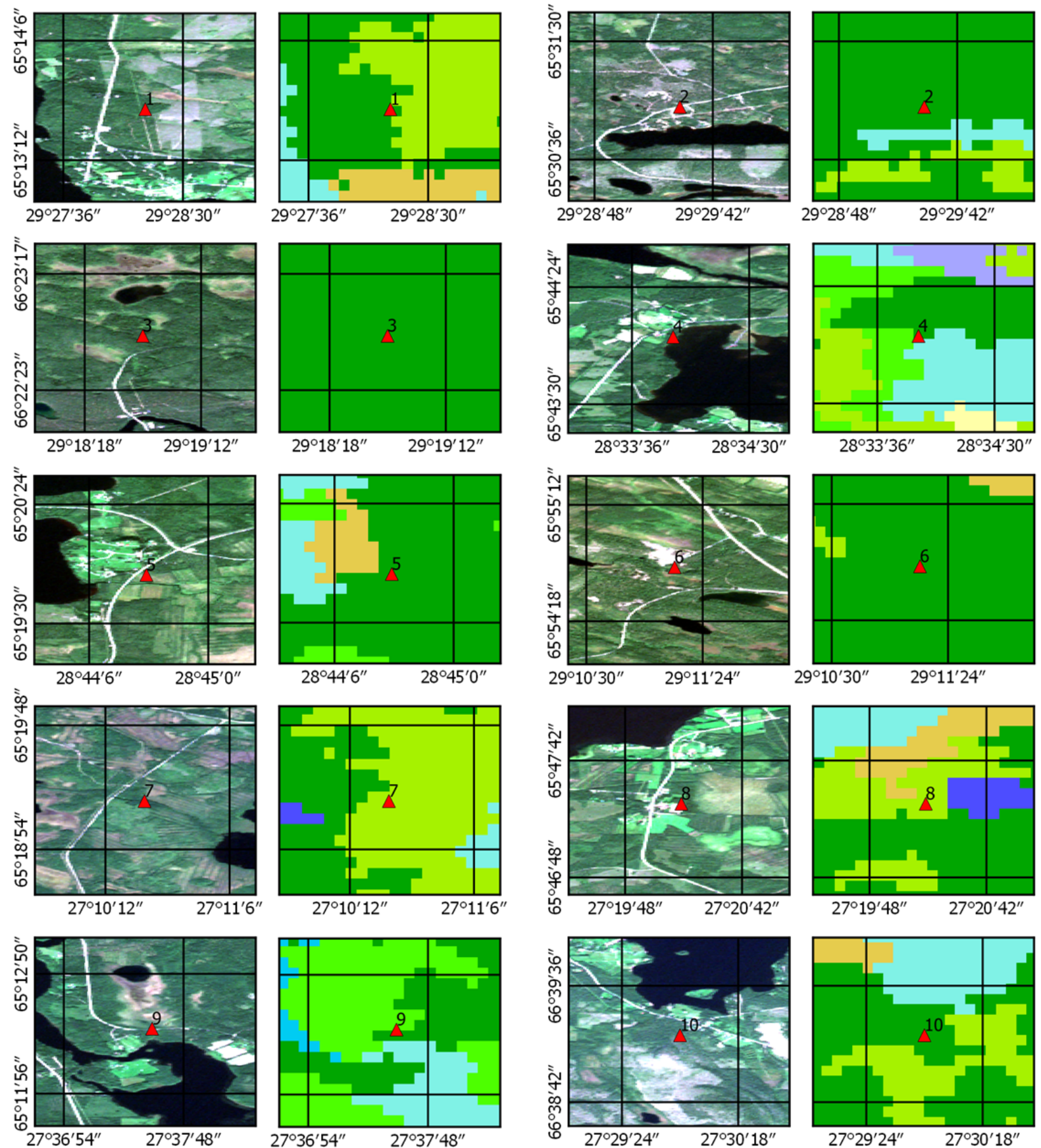

Fig. 3. From left to right, from top to bottom, details of cloud and snow-free Sentinel-2 MSI Natural Colors and 2012-Corine map around the ten locations of in-situ SWE measurements (denotes as red triangles with the corresponding location number used throughout the paper). The colors in the Corine map denote: a) black green - coniferous forest; b) bright green - transitional woodland-shrub; c) yellowbrown - land principally occupied by agriculture with significant areas of natural vegetation; d) cyan - water bodies; e) mauve violet inland marshed; f) dark violet - peat bogs.

the modelling of phase delay in atmosphere is not correct, also the estimate of $\triangle \mathrm{SWE}$ is not correct. The phase delay in atmosphere is computed using the output of the Weather Research and Forecast (WRF) model. This is a next-generation mesoscale numerical weather prediction system used in a wide range of meteorological applications across scales ranging from meters to thousands of kilometers. Four one-way nested domains at 27-, 9-, 3-, and 1-km horizontal grid resolutions were defined. The initial time-dependent boundary conditions are derived from the latest European Centre for Medium Range Weather Forecasting (ECMWF)/ERA-Interim global atmospheric reanalysis (1989-present). The top of the atmosphere in the model is located at the $10-\mathrm{hPa}$ level, approximately corresponding to a height of about $30 \mathrm{~km}$; a total of 50 vertical layers that are unevenly spaced were used, and the lowest model layer is about $60 \mathrm{~m}$ thick. The model is used to simulate a 3-D field with the vertical profiles of the atmosphere's parameters (temperature, pressure, water vapor fraction, geopotential, and precipitable water vapor) generated over a $1 \mathrm{~km} \times 1 \mathrm{~km}$ grid. The initial and time-dependent conditions were derived from the European Centre for Medium Range Weather ForecastsERA Interim reanalysis. The grid resolution of the ERAInterim data is $0.75^{\circ} \times 0.75^{\circ}$, with a total of 37 pressure levels. Simulations were conducted starting $12 \mathrm{~h}$ before SAR 


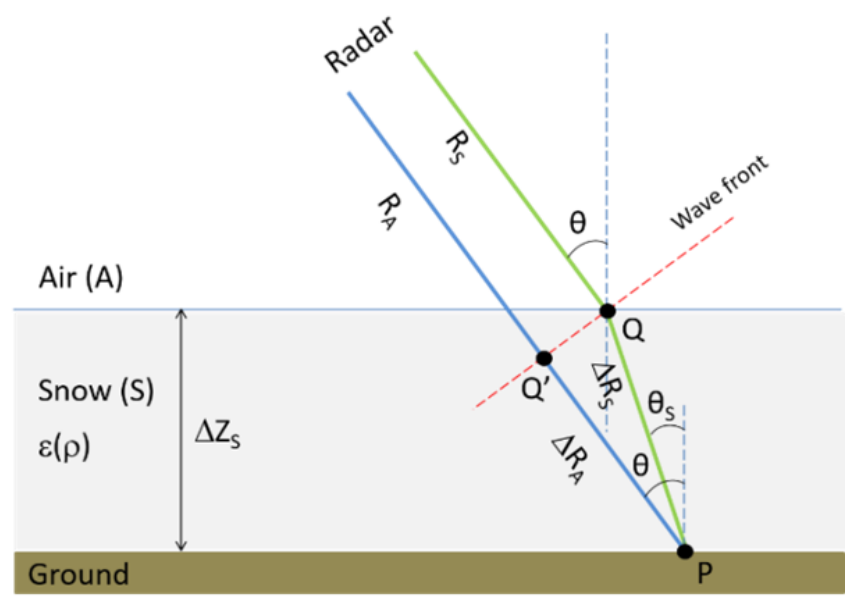

Fig. 4. Schematic depiction of the optical path delay of SAR microwaves when a uniform snow layer is crossed giving rise to the interferometric phase due to SWE $\left(\Delta \phi_{S}\right) . \mathrm{R}_{\mathrm{A}}$ denotes the SAR range in air (without snow) and $R_{S}$ is the SAR range when a uniform snow layer of $\Delta Z_{S}$ depth is intersected (Leinss et al., 2015).

acquisition time. Delays computed using a ray-tracing procedure were interpolated over the geolocated InSAR grid (Nico, 2002). These quantities were used to compute the 3D field of atmosphere refractivity at the acquisition times of the two Sentinel-1 SAR images used to generate a SAR interferogram. The phase delay at a given time has been obtained by integrating the phase delay along the SAR line-ofsight using a ray tracing procedure. The difference of phase delays at the acquisition times of master and slave SAR images, mapped from WRF longitude/latitude grid to Sentinel-1 range/azimuth grid, provides the phase screen used to correct atmosphere propagation effects in the SAR interferogram. More details about WRF schemes used to model land-surface, the turbulence in the planetary boundary layer, the atmosphere convection and the cloud microphysics, as well as the accuracy WRF-based maps of phase delay in atmosphere can be found in Mateus et al. (2015) and references therein. The synthetic interferograms corresponding to the atmospheric phase delays were then obtained as the difference between the atmospheric phase delays computed at the acquisition times of the master and slave SAR images.

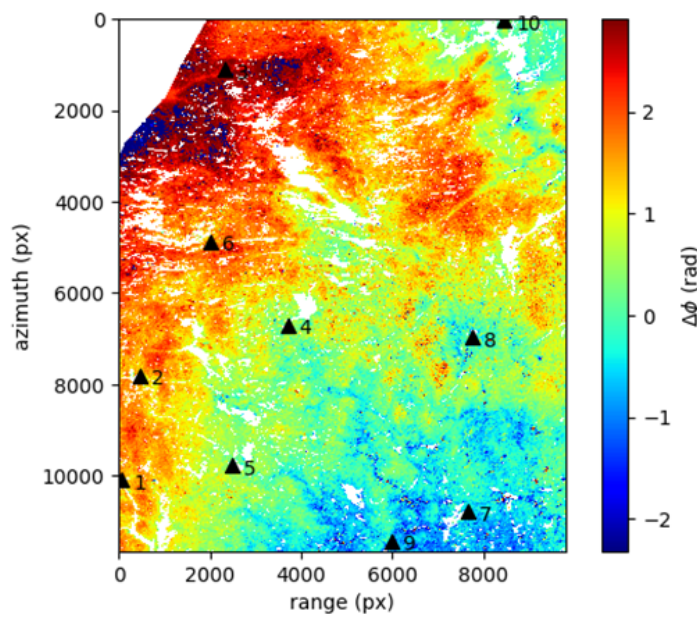

\section{RESULTS}

In this section, the results which have been obtained over the study area are summarized. Figure 5 shows the two interferograms which have been generated, after the topographic phase correction. They correspond to the time intervals between December 29, 2015 and January 10, 2016 and between January 10, 2016 and January 22, 2016. Lakes and other large water surfaces have been masked out and appear as white pixels. Also the portion of the interferogram falling on the Russian territory has been mask out. It appears as a white area on the top-left of the interferogram. More Sentinel-1 SAR images have been acquired in the time interval for which in-situ measurements were available. However, interferograms built with those images showed a poor interferometric coherence and for this reason they have not been used in this study. Figure 6 shows the two synthetic interferograms related to the temporal change of propagation properties in atmosphere. The atmospheric artifacts were mitigated by removing the synthetic image of atmospheric phase delay from the corresponding SAR interferogram. Figure 7 shows the interferograms, after the correction of topographic and atmospheric phase contributions. Phase measurements have been unwrapped both in time and space using in-situ SWE measurements to know the expected true absolute phase values in a few points and to detect possible phase unwrapping errors. We need these in-situ measurements as a reference, in the same way as the knowledge of stable reference points is needed in the geodetic applications of SAR interferometry. The in-situ $\triangle$ SWE measurements at the three locations 6, 9 and 10 have been used to calibrate the unwrapped phase.

Calibration procedure would require just one in-situ SWE measurement. However, we used three in-situ measurements to get some redundancy, in an attempt to reduce the probability of getting a biased calibration and to identify a spatial trend.

The final result is reported in Figure 8 which displays the two maps with the temporal variation of SWE occurred between the master and slave acquisition times.

The InSAR maps of $\triangle$ SWE have been assessed using the in-situ measurements at the remaining in-situ locations not used for the calibration of the unwrapped interferometric phase. For each location, a $3 \times 3$ window around its position on the InSAR $\triangle \mathrm{SWE}$ map has been used to identify pixels on the map. The $\triangle$ SWE values estimated at those pixels have been used to compute the mean value to be compared with the in-situ measurement at the location. Pixels corresponding to water

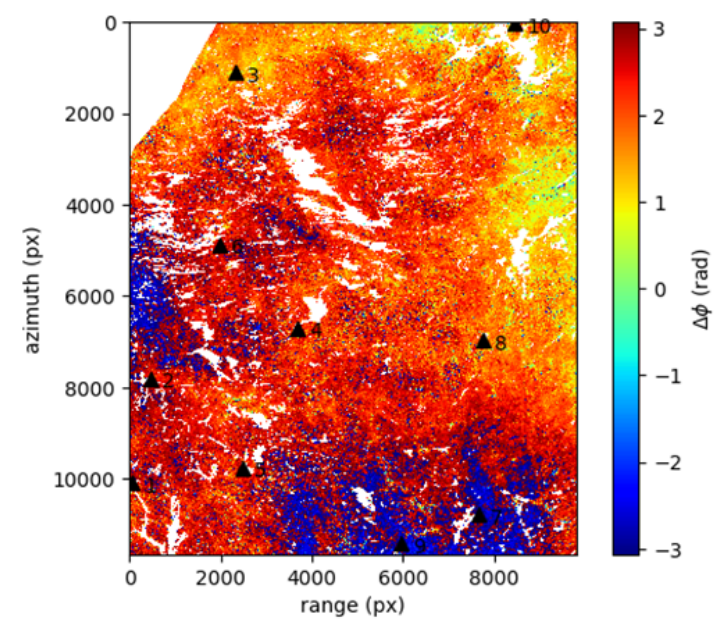

Fig. 5. Interferograms after topographic correction. (a) December 29, 2015-January 10, 2016. (b) January 10, 2016-January 22, 2016. 

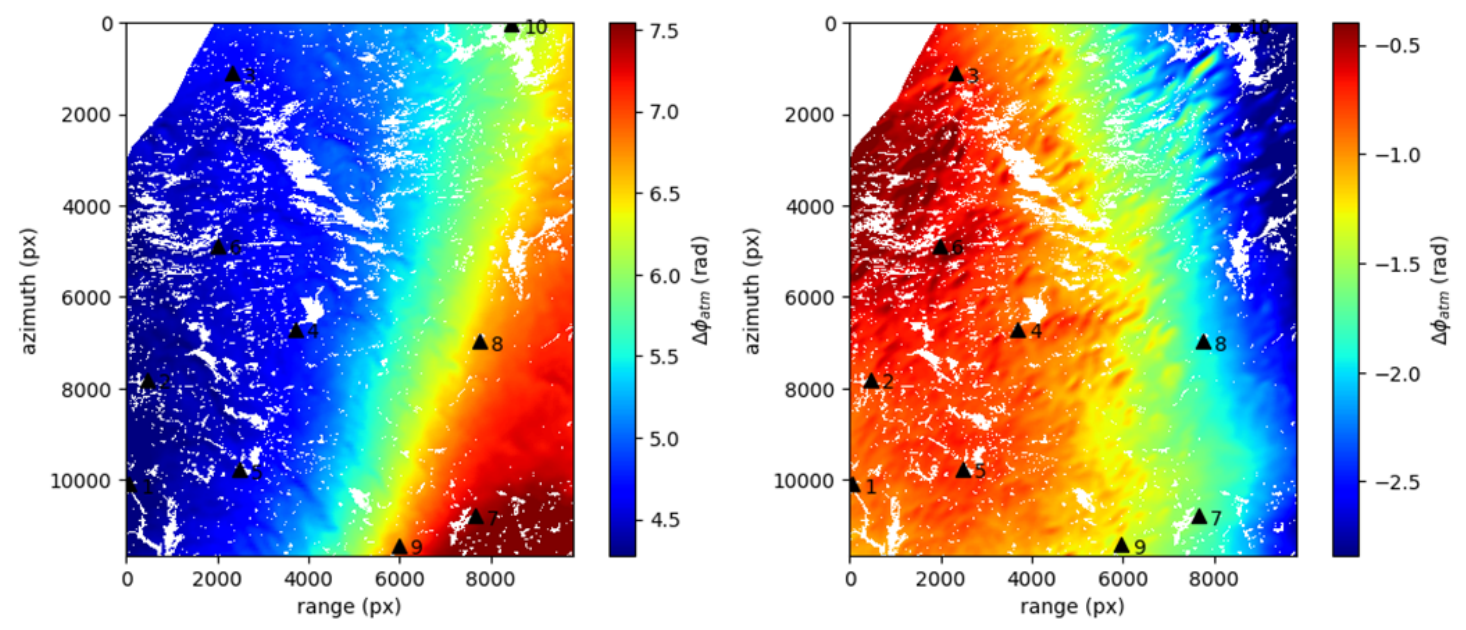

Fig. 6. Synthetic interferograms of atmospheric phase delay $\left(\Delta \phi_{\text {atm }}\right)$ derived from WRF model. (a) $\Delta \phi_{\text {atm }}$ between December 29,2015 and January 10, 2016. (b) $\Delta \phi_{\text {atm }}$ between January 10, 2016 and January 22, 2016.
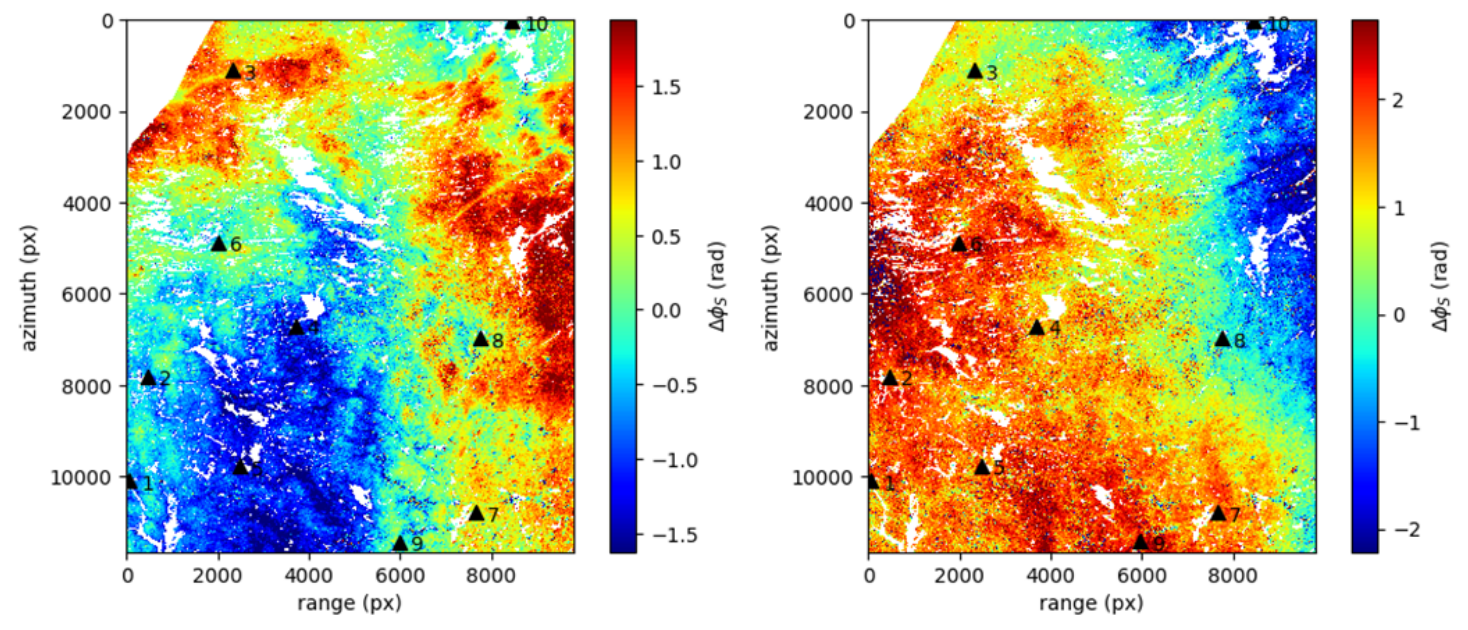

Fig. 7. Interferograms after topographic and atmospheric corrections. (a) December 29, 2015-January 10, 2016. (b) January 10, 2016January 22, 2016.
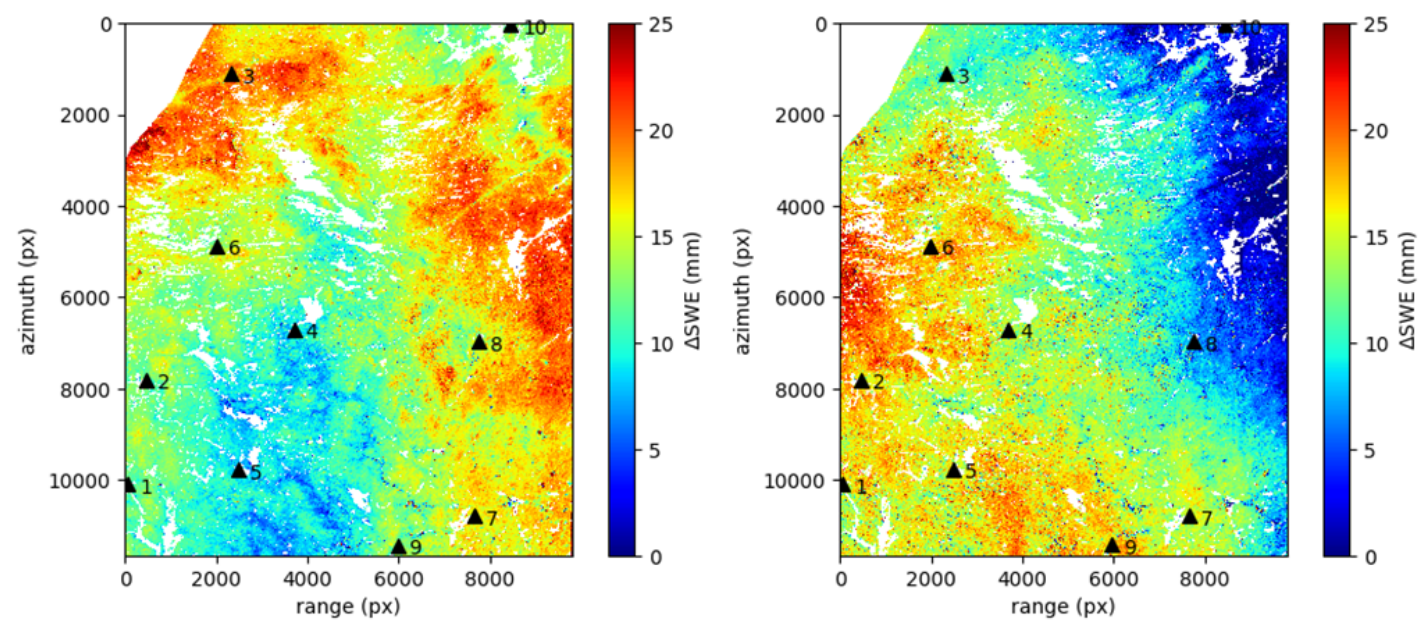

Fig. 8. $\triangle$ SWE maps derived by SAR interferometry with overlaid the location of in-situ measurements of SWE (black triangles). (a) $\triangle \mathrm{SWE}$ between December 29, 2015 and January 10, 2016. (b) $\triangle$ SWE between January 10, 2016 and January 22, 2016.

surfaces, if falling within the $3 \times 3$ window, have not been taken for the computation of the mean values. Tables 2 and 3 summarize the comparison between the InSAR estimates and the $\triangle \mathrm{SWE}$ values derived from the in-situ measurements. It is worth noting that the in-situ $\triangle \mathrm{SWE}$ estimates at the acquisition times of Sentinel-1 images, reported in these tables have been obtained by a 2 nd-order polynomial regression. 
Table 2. In-situ $\triangle$ SWE measurements, $\triangle$ SWE InSAR derived and their difference. (December 29, 2015-January 10, 2016).

\begin{tabular}{|c|c|c|c|c|}
\hline $\begin{array}{c}\text { In-situ } \\
\text { location }\end{array}$ & $\begin{array}{c}\Delta \mathrm{SWE}_{\text {in-situ }} \\
(\mathrm{mm})\end{array}$ & $\begin{array}{c}\Delta \mathrm{SWE}_{\text {InSAR }} \\
(\mathrm{mm})\end{array}$ & $\begin{array}{c}\text { Difference } \\
(\mathrm{mm})\end{array}$ & $\begin{array}{c}\text { Difference } \\
(\%)\end{array}$ \\
\hline 1 & 12.6 & 7.6 & 5.0 & 39.4 \\
\hline 2 & 14.3 & 6.6 & 7.7 & 53.8 \\
\hline 3 & 14.8 & 13.7 & 1.1 & 7.5 \\
\hline 4 & 19.2 & 5.2 & 13.9 & 72.6 \\
\hline 5 & 17.6 & 5.6 & 12.0 & 68.4 \\
\hline 7 & 6.6 & 12.0 & -5.4 & -81.5 \\
\hline 8 & 11.1 & 11.3 & -0.2 & -2.0 \\
\hline \multirow{2}{*}{} & & mean & 4.9 & 23.1 \\
\cline { 2 - 5 } & std & 6.9 & 53.8 \\
\cline { 3 - 5 } & & & \\
\end{tabular}

Table 3. In-situ $\triangle$ SWE measurements, $\triangle$ SWE InSAR derived and their difference. (January 10, 2016-January 22, 2016).

\begin{tabular}{|c|c|c|c|c|}
\hline $\begin{array}{c}\text { In-situ } \\
\text { location }\end{array}$ & $\begin{array}{c}\Delta \mathrm{SWE}_{\text {in-situ }} \\
(\mathrm{mm})\end{array}$ & $\begin{array}{c}\Delta \mathrm{SWE}_{\text {InSAR }} \\
(\mathrm{mm})\end{array}$ & $\begin{array}{c}\text { Difference } \\
(\mathrm{mm})\end{array}$ & $\begin{array}{c}\text { Difference } \\
(\%)\end{array}$ \\
\hline 1 & 17.0 & 16.4 & 0.7 & 3.9 \\
\hline 2 & 18.8 & 14.3 & 4.5 & 24.1 \\
\hline 3 & 14.8 & 10.3 & 4.5 & 30.1 \\
\hline 4 & 23.6 & 14.0 & 9.6 & 40.6 \\
\hline 5 & 17.6 & 14.9 & 2.7 & 15.2 \\
\hline 7 & 6.6 & 14.5 & -7.9 & -120.2 \\
\hline 8 & 11.1 & 7.8 & 3.3 & 30.1 \\
\hline & & mean & 2.5 & 3.4 \\
\cline { 2 - 5 } & std & 5.3 & 56.8 \\
\hline
\end{tabular}

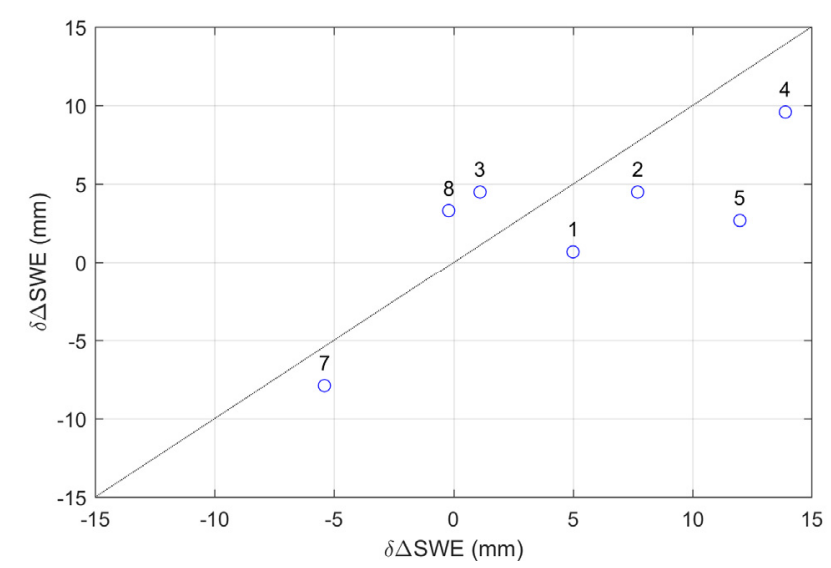

Fig. 9. Scatter plot of $\delta \Delta S W E\left(\Delta S W E_{\text {InSAR }}-\Delta S W E_{\text {in-situ }}\right)$ for the period of the first interferogram vs. $\delta \Delta \mathrm{SWE}$ for the period of the second interferogram.

The differences between the two values are also reported, both as a value in millimeters and in percentage with the respect to the in-situ measurements. The comparison shows a reasonable match between InSAR estimates and in-situ measurements of $\triangle \mathrm{SWE}$ with an average accuracy of about $6 \mathrm{~mm}$. Figure 9 shows the comparison of differences between InSAR estimates of $\triangle \mathrm{SWE}$ and the corresponding quantities obtained by interpolating the in-situ measurements. The almost linear trend indicates that quality of the InSAR estimates of $\triangle \mathrm{SWE}$ does not depend on the interferogram that has been used. When the difference of the $\triangle \mathrm{SWE}$ estimate with respect to the in-situ measurement is almost the same for the two interferograms, it means that this difference depends on the land cover as explained before. However, the accuracy of the $\triangle \mathrm{SWE}$ could also depend on the interferometric coherence that could change from one interferogram to the other as in the case of in-situ measurement at location 5 .

\section{DISCUSSION AND CONCLUSIONS}

In this paper a new methodology to estimate temporal changes of $\triangle \mathrm{SWE}$ has been presented, based on the SAR interferometry technique and the use of a NWM to model and remove the phase contribution due to propagation in the atmosphere. Sentinel-1 SAR images with spatial resolution of $20 \mathrm{~m}$ have been used. The comparison with an independent set of $\triangle$ SWE estimates derived by in-situ measurements shows that the proposed methodology can attain an accuracy of about 6 $\mathrm{mm}$ which is in agreement with the root-mean-square errors obtained using a ground-based radar working at frequencies of $10 \mathrm{GHz}$ and $16 \mathrm{GHz}$ (Leinss et al., 2015). The $\Delta$ SWE estimates have an error between 5 and $15 \mathrm{~mm}$, for temporal variations of the SWE up to $200 \mathrm{~mm}$. It was found that the interferometric coherence was preserved for almost a month in case of dry snow. In contrast, the presence of wet snow shortened the decorrelation time to a few hours (Leinss et al., 2015). This property of the interferometric coherence has been confirmed by our results, even if they have been obtained using a spaceborne C-band SAR sensor instead of a ground-based Ku-band radar. In fact, we found that between December and January, when it can be supposed to have dry snow, the interferometric coherence of SAR images acquired with a temporal baseline of 12 days was high and allowed to estimate the $\triangle$ SWE over almost the whole SAR image except for lakes and dense forests where some decorrelation effect has been observed.

Concerning SWE measurement provided by other spaceborne sensors, it has been demonstrated that the assimilation of passive microwave data in a semi-empirical radiative transfer model can provided error values of 15 to $40 \mathrm{~mm}$ in the estimation of the SWE (Pulliainen and Hallikainen (2001); Pulliainen (2006)). A similar error has been reported by Takala et al. (2011) who estimated the SWE over Russia, Finland and Canada with an error below $40 \mathrm{~mm}$. However, the spatial resolution of radiometer is by far lower than that which can be attained by spaceborne SAR sensors.

Other methods combining remotely sensed snow cover images with a spatially distributed snowmelt model were characterized by a root-mean-square error of about $250 \mathrm{~mm}$ when validated against snow survey observations (Guan et al., 2013).

Based on these results, we conclude that SAR interferometry could be a promising technique to get high-resolution maps of $\triangle \mathrm{SWE}$, updated up to every six days if Sentinel-1A\&B data are used, also in areas where few in-situ measurements are available to invert microwave backscattering models. If a reference image is taken in a period when snow is not covering the terrain surface (e.g. summer season), a time series of SWE estimates can be recovered from the $\triangle \mathrm{SWE}$ maps provided by SAR interferometry. The temporal updating of $\triangle \mathrm{SWE}$ maps can be further reduced to less than six days if the $\triangle \mathrm{SWE}$ measurements obtained by SAR images acquired along different orbits are merged. The interferometric coherence is a critical point since it can hinder the estimate of $\triangle \mathrm{SWE}$ in areas covered by vegetation or when large snowfalls completely change the surface morphology so destroying the interferometric coherence. The lack of coherence can reduce the number of SAR interferograms that can be used to get maps of $\triangle \mathrm{SWE}$. From the physical point of view, the proposed methodology requires a dry snow in order to allow the radar signal to penetrate the snow layer without being absorbed or reflected and the assumption that snow density is constant over the scene. This last assumption can be relaxed if polarimetric SAR images are also used. 
To summarize, we found that the proposed methodology, relying on Sentinel-1 interferograms, with a 6-day temporal baseline, could map SWE also over regions covered by shrubs or low vegetation. The main advantage of this methodology with respect to other approaches to estimate $\triangle \mathrm{SWE}$ based on SAR amplitude is that a small number of in-situ measurements of SWE are needed.

Acknowledgements. This work was supported in part by the Fundação para a Ciência e a Tecnologia (FCT), Portugal, under $\mathrm{PhD}$ Grant PD/BD/106021/2014 and by FCT-Instituto Dom Luiz under Project UID/GEO/50019/2013. Authors acknowledge being a part of the networks supported by the COST Action TD1403 "Big Data Era in Sky and Earth Observation" and the COST Action ES1404 "A European network for a harmonised monitoring of snow for the benefit of climate change scenarios, hydrology and numerical weather prediction". MG acknowledges support from the ERC Advanced Grant No. 320773, and the Russian Foundation for Basic Research, project nos. 16-05-00004 and 16-07-01072. Research at the Ural Federal University is supported by the Act 211 of the Government of the Russian Federation, agreement No 02.A03.21.0006.

\section{REFERENCES}

Bavera, D., De Michele, C., 2009. Snow water equivalent estimation in the Mallero basin using snow gauge data and MODIS images and fieldwork validation. Hydrological Processes, 23, 14, 1961-1972.

Guan, B., Molotch, N.P., Waliser, D.E., Jepsen, S.M., Painter, T.H., Dozier, J., 2013. Snow water equivalent in the Sierra Nevada: Blending snow sensor observations with snowmelt model simulations, Water Resour. Res., 49, 5029-5046.

Guneriussen, T., Hogda, K., Johnsen, H., Lauknes, I., 2001. InSAR for estimation of changes in snow water equivalent of dry snow. IEEE Transactions on Geoscience and Remote Sensing, 39, 10, 2101-2108.

Krajči, P., Danko, M., Kostka, Z., Holko, L., 2016. Experimental measurements for improved understanding and simulation of snowmelt events in the Western Tatra Mountains, J. Hydrol. Hydromech., 64, 4, 316-328.

Leinss, S., Wiesmann, A., Lemmetyinen, J., Hajnsek, I., 2015. Snow Water Equivalent of Dry Snow Measured by Differential Interferometry. IEEE Journal of Selected Topics in Applied Earth Observations and Remote Sensing, 8, 8, 37733790.

Leppänen, L., Kontu, A., Hannula, H.-R., Sjöblom, H., Pulliainen, J., 2016. Sodankylä manual snow survey program. Geoscientific Instrumentation, Methods and Data Systems, 5, 163-179.

Longepe, N., Allain, S., Ferro-Famil, L., Pottier, E., Durand, Y., 2009. Snowpack characterization in mountainous regions using C-band SAR data and a meteorological model. IEEE Transactions on Geoscience and Remote Sensing, 47, 2, 406-418.

Malnes, E., Guneriussen, T., 2002. Mapping of snow covered area with Radarsat in Norway. In: Proc. Geoscience and Remote Sensing Symposium IGARSS '02, Vol.1. IEEE, Toronto, Ontario, Canada, pp. 683-685.
Mateus, P., Nico, G., Catalão, J., 2010. Interpolating MERIS and GPS measurements of precipitable water vapor (PWV) to estimate atmospheric phase delay maps. In: Proc. SPIE, Remote Sensing of Clouds and the Atmosphere XV, 7827.

Mateus, P., Nico, G., Catalão, J., 2015. Uncertainty assessment of the estimated atmospheric delay obtained by a Numerical Weather Model (NMW). IEEE Transactions on Geoscience and Remote Sensing, 53, 6710-6717.

Nagler, T., Rott, H., 2000. Retrieval of wet snow by means of multitemporal SAR data. IEEE Transactions on Geoscience and Remote Sensing, 38, 2, 754-765.

Nico, G, 2002. Exact closed-form geolocation for SAR interferometry. IEEE Transactions on Geoscience and Remote Sensing, 40, 1, 220-222.

Nico, G., Tome, R., Catalão, J., Miranda, P.M., 2011. On the Use of the WRF model to mitigate tropospheric phase delay effects in SAR interferograms. IEEE Transactions on Geoscience and Remote Sensing, 49, 4970-4976.

Pulliainen, J., 2006. Mapping of snow water equivalent and snow depth in boreal and sub-arctic zones by assimilating space-borne microwave radiometer data and ground-based observations. Remote Sensing of Environment, 101, 257269.

Pulliainen, J., Hallikainen, M., 2001. Retrieval of regional snow water equivalent from spaceborne passive microwave observations. Remote Sensing of Environment, 75, 1, 76-85.

Pivot, F.C., 2012. C-band SAR imagery for snow-cover monitoring at treeline, Churchill, Manitoba, Canada. Remote Sensing, 4, 7, 2133.

Rott, H., Duguay, C., Etchevers, P., Essery, R., Hajnsek, I., Macelloni, G., Malnes, E., Pulliainen, J., 2012a. Report for Mission Selection: CoReH20. Technical Report. European Space Agency, Nordwijk, The Netherlands.

Rott, H., Cline, D.W., Duguay, C., Essery, R., Etchevers, P., Hajnsek, I., Kern, M., Macelloni, G., Malnes, E., Pulliainen, J., Yueh, S.H., 2012b. CoReH2O, a dual frequency radar mission for snow and ice observations. In: Proc. Geoscience and Remote Sensing Symposium (IGARSS 2012). IEEE, Toronto, Ontario, Canada, pp. 5550-5553.

Shi, J., Dozier, J., 2000a. Estimation of snow water equivalence using SIR-C/X-SAR. I. Inferring snow density and subsurface properties. IEEE Transactions on Geoscience and Remote Sensing, 38, 6, 2465-2474.

Shi, J., Dozier, J., 2000b. Estimation of snow water equivalence using SIR-C/X-SAR. II. Inferring snow depth and particle size. IEEE Transactions on Geoscience and Remote Sensing, 38, 6, 2475-2488.

Sun, S., Che, T., Wang, J., Li, H., Hao, X., Wang, Z., Wang, J., 2015. Estimation and analysis of snow water equivalents based on C-band SAR data and field measurements. Arctic, Antarctic, and Alpine Research, 47, 2, 313-326.

Takala, M., Luojus, K., Pulliainen, J., Derksen, C., Lemmetyinen, J., Kärnä, J.-P., Koskinen, J., Bojkov, B., 2011. Estimating northern hemisphere snow water equivalent for climate research through assimilation of space-borne radiometer data and ground-based measurements. Remote Sensing of Environment, 115, 3517-3529.

Received 30 June 2017 Accepted 26 October 2017 